\title{
Intracranial hypertension following sacrifice of occipital and marginal sinuses during posterior fossa decompression for Chiari I malformation: case report
}

\author{
Pierre-Olivier Champagne, MD, FRCSC, ${ }^{1}$ Florence Cayouette, MD, ${ }^{2}$ Anne Lortie, MD, ${ }^{2}$ \\ Jean-Claude Decarie, MD, ${ }^{3}$ and Alexander G. Weil, MD, FRCSC ${ }^{1}$
}

\begin{abstract}
Departments of ${ }^{1}$ Pediatric Neurosurgery, ${ }^{2}$ Neurology, and ${ }^{3}$ Radiology, Centre Hospitalier Universitaire Sainte-Justine, Université
\end{abstract} de Montréal, Montreal, Quebec, Canada

\begin{abstract}
The occipital and marginal sinuses, when present, must be sacrificed in order to open the dura in most posterior fossa surgeries in the pediatric population, including posterior fossa decompression for Type I Chiari malformation (CM-I) with duraplasty. Apart from the immediate risk of hemorrhage, the voluntary occlusion of this structure is almost universally well tolerated. The authors report a case of intracranial hypertension following the sacrifice of occipital and marginal sinuses following posterior fossa decompression with duraplasty for CM-I. The specific draining pattern variant of the occipital and marginal sinuses leading to this complication as well as avoidance and management strategies of this condition are discussed.
\end{abstract}

https://thejns.org/doi/abs/10.3171/2018.7.PEDS18237

KEYWORDS intracranial hypertension; occipital sinus; venous hypertension; craniotomy; vascular disorders; Chiari

$\mathrm{S}$ UBOCCIPITAL craniotomy represents the standard means of access to the posterior fossa. The occipital sinus, a midline structure present mainly in the pediatric population, must usually be sacrificed to allow for optimal dura opening and potential duraplasty. ${ }^{6}$ In posterior fossa decompression, most surgeons perform duraplasty in addition to suboccipital craniectomy and $\mathrm{C} 1$ laminectomy. Sacrifice of the occipital or marginal sinus during surgery usually bears no clinical impact. We report a new complication secondary to occipital and marginal sinus sacrifice in a patient undergoing a posterior fossa craniectomy and duraplasty for a Type I Chiari malformation (CM-I). We discuss the pathophysiology and impact of this condition as well as its management avenues.

\section{Case Report}

A 15-year-old girl presented with a 10-month history of truncular paresthesias affecting bilaterally the upper torso, scapula, and neck. The remainder of the neurological examination findings were normal. Brain and cervical spine MRI revealed CM-I with cerebellar displacement of $7 \mathrm{~mm}$ below the foramen magnum and the presence of hydromyelia spanning almost the entire length of the spinal cord (Fig. 1). The patient underwent posterior fossa decompression. The posterior fossa and foramen magnum were decompressed via a suboccipital craniectomy and a laminectomy of the posterior arch of $\mathrm{Cl}$. Dura opening and closure with a large duraplasty allowed for an optimal decompression. Of note, patent occipital and marginal sinuses previously viewed on brain MRI were carefully sacrificed intraoperatively using hemoclips upon dural opening with no particular concern nor any abnormal bleeding. The estimated blood loss during surgery was less than 150 $\mathrm{ml}$. The patient's condition evolved well in the immediate postoperative period, and she returned home on the fifth postoperative day.

The patient presented 10 days after surgery with severe headaches, vomiting, and new-onset horizontal diplopia. Neurological examination revealed the presence of a bilateral sixth cranial nerve palsy and bilateral papilledema, which were both confirmed by a neuro-ophthalmologist. No visual field or visual acuity anomaly was detected. Brain MRI showed good decompression of the posterior fossa and foramen magnum, reduction in size of the hy-

ABBREVIATIONS CM-I = Type I Chiari malformation.

SUBMITTED April 21, 2018. ACCEPTED July 10, 2018

INCLUDE WHEN CITING Published online September 21, 2018; DOI: 10.3171/2018.7.PEDS18237. 

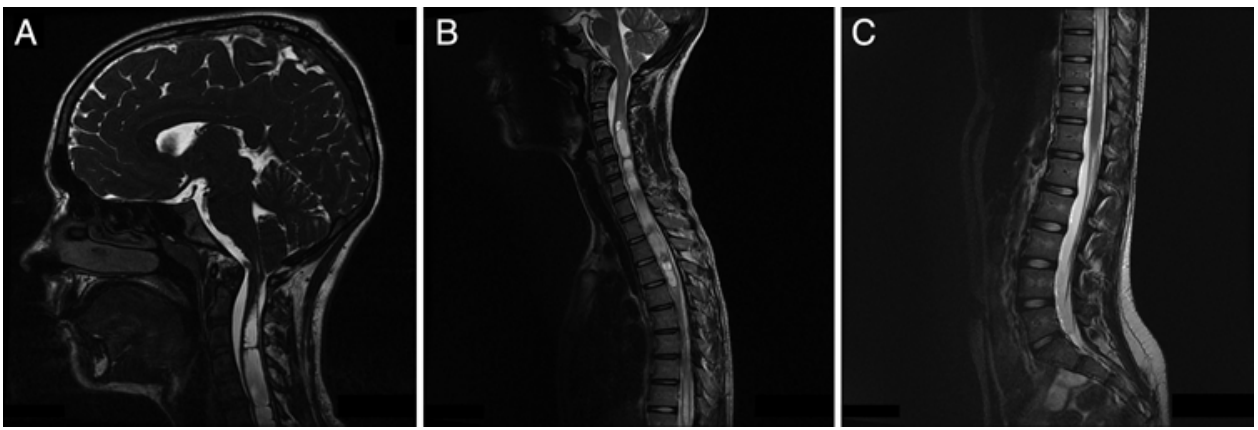

FIG. 1. Preoperative T2-weighted MRI. A: Sagittal image of the head showing CM-I and the superior extension of the hydromyelia. B: Sagittal image of the thoracic spine showing hydromyelia spanning the entire thoracic cord. C: Sagittal image of the lumbar spine showing hydromyelia reaching the conus medullaris.

dromyelia, and normal ventricles (Fig. 2). Several signs of raised intracranial pressure were observed, including vertical tortuosity of the optic nerves. Postoperative MRI confirmed absence of occipital and marginal sinuses (Fig. 3). On reassessment of perioperative MRI, we observed a very prominent occipital sinus, which drained directly through an equally prominent marginal sinus into the sigmoid sinus and jugular vein bilaterally (Fig. 4). Both transverse sinuses were relatively hypoplastic. The working diagnosis at this time (i.e., when the patient returned to the hospital with these new symptoms) was intracranial hypertension caused by diminished venous drainage secondary to occipital and marginal sinus sacrifice. A lumbar puncture confirmed the diagnosis of intracranial hypertension with an opening pressure of $47 \mathrm{~cm} \mathrm{H}_{2} \mathrm{O}(34 \mathrm{~mm}$ $\mathrm{Hg}$ ). CSF analysis revealed no anomalies (glucose 2.78 $\mathrm{mmol} / \mathrm{L}$, protein $0.44 \mathrm{~g} / \mathrm{L}$, white blood cells $139 \times 10^{6} / \mathrm{L}$, red blood cells $7 \times 10^{6} / \mathrm{L}$, neutrophils 0.18 , and lymphocytes 0.71 ). Unfortunately, no preoperative ophthalmological examination had been performed, because preexisting pseudotumor cerebri was not suspected on a clinical basis or based on body habitus (body mass index [BMI] 20.3, 55th percentile). The patient's symptoms were alleviated after the lumbar puncture and the decision was made to manage her condition medically with diuretic therapy (acetazolamide therapy, $250 \mathrm{mg}$ TID). On follow-up 2 months after the initial surgery, the patient was still well, taking acetazolamide, with resolution of headaches, no vomiting, resolution of the sixth cranial nerve palsy, and resolution of the papilledema documented on serial neuro-ophthalmological examinations. At 4-month follow-up, symptoms had resolved, and she had been completely weaned from acetazolamide therapy. Regarding Chiari symptoms, the paresthesia had resolved completely at 4 months, with only slight hyperreflexia of the lower left extremity remaining. An MRI study performed at that time showed diminution in size of the syrinx. At last 12-month followup, all symptoms have resolved and there is no recurrence of symptoms or signs of intracranial hypertension.

\section{Discussion}

We report, to our knowledge, the first case of intracranial hypertension related to occipital and marginal sinus sacrifice during posterior fossa surgery in a pediatric patient. The occipital sinus is a midline dural structure running in the falx cerebelli, joining the posterior confluence of the marginal sinus and the torcula. ${ }^{7}$ Embryologically, the occipital sinus has been visualized as early as the third gestational month, ${ }^{5}$ and its development follows a course of fusion and obliteration of midline dural channels originating from the torcula. ${ }^{11}$ Cadaveric and imaging studies have shown the presence of occipital sinuses in every newborn ${ }^{3}$ and a declining incidence of this structure with age. ${ }^{11}$ One hypothesis explaining the regression of this structure with age states that with erect posture, the main intracranial venous drainage shifts from the internal jugular veins to the vertebral venous system, diminishing
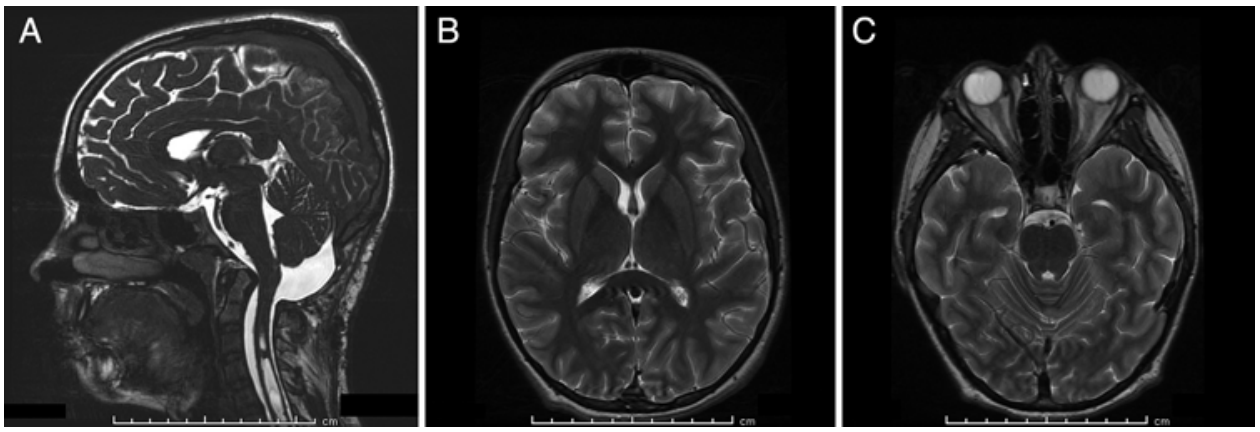

FIG. 2. Postoperative T2-weighted MRI. A: Sagittal image showing adequate decompression of the craniocervical junction. B and C: Axial images showing ventricles of normal size. 

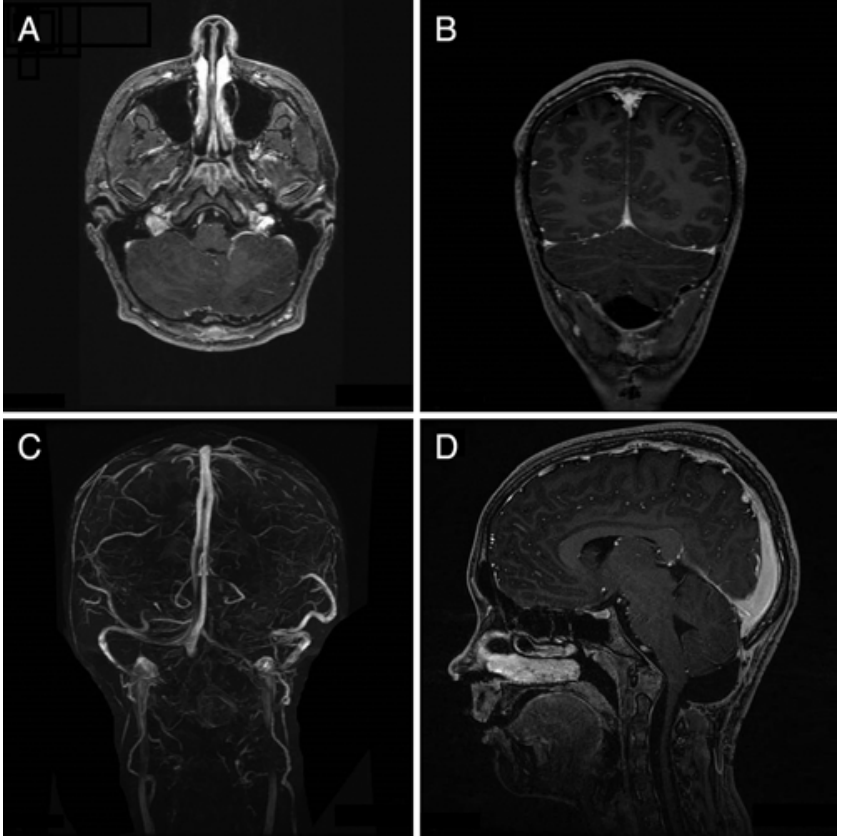

FIG. 3. Postoperative MR images showing absence of occipital and marginal sinuses and slightly hypoplastic transverse sinuses. A, B, and D: Gd-enhanced T1-weighted axial (A), coronal (B), and sagittal (D) images. C: $3 D$ reconstruction of venous drainage from an MR angiography (MRA)-MR venography (MRV).

the flow into the occipital sinus. ${ }^{11}$ Variants of the occipital sinus have been described, such as multiples sinuses ${ }^{3}$ and an occipital sinus draining into the transverse sinus ${ }^{8}$ or into the sigmoid sinus. ${ }^{2}$ The occipital sinus usually drains from the torcula toward the marginal sinus. ${ }^{2}$ The presence of other exit pathways from the torcula-i.e., through the transverse sinus-explains why the sacrifice of the occipital sinus usually has no clinical consequence. However, in some cases, such as in patients with unilateral or bilateral transverse sinus hypoplasia,,$^{2,9,10}$ the occipital sinus can represent the main venous outflow from the torcula. We suggest that this was the situation in our case.

We report an unusual anatomical variant of the occipital sinus, with the sinus draining bilaterally into the jugular bulbs through the marginal sinus. The uniqueness of this case lies in the fact that although the transverse and sigmoid sinuses were still patent and of almost normal caliber bilaterally, the occipital sinus was clearly dominant compared to the transverse sinus and, as proved by the clinical impact of its sacrifice, represented a major venous outflow from the torcula in our patient. We believe that it is this impaired venous outflow that caused widespread venous congestion, causing intracranial hypertension both through the congestion itself and through impaired CSF reabsorption. It has already been suggested that in cases in which the occipital sinus is suspected to be the dominant drainage it should be left intact. ${ }^{4}$ We thus suggest that occipital sinus and marginal sinus size along with the drainage pattern into the jugular bulbs should be taken into consideration when establishing dominance.

The clinical relevance of dominant occipital sinus occlusion has mainly been presented hypothetically until
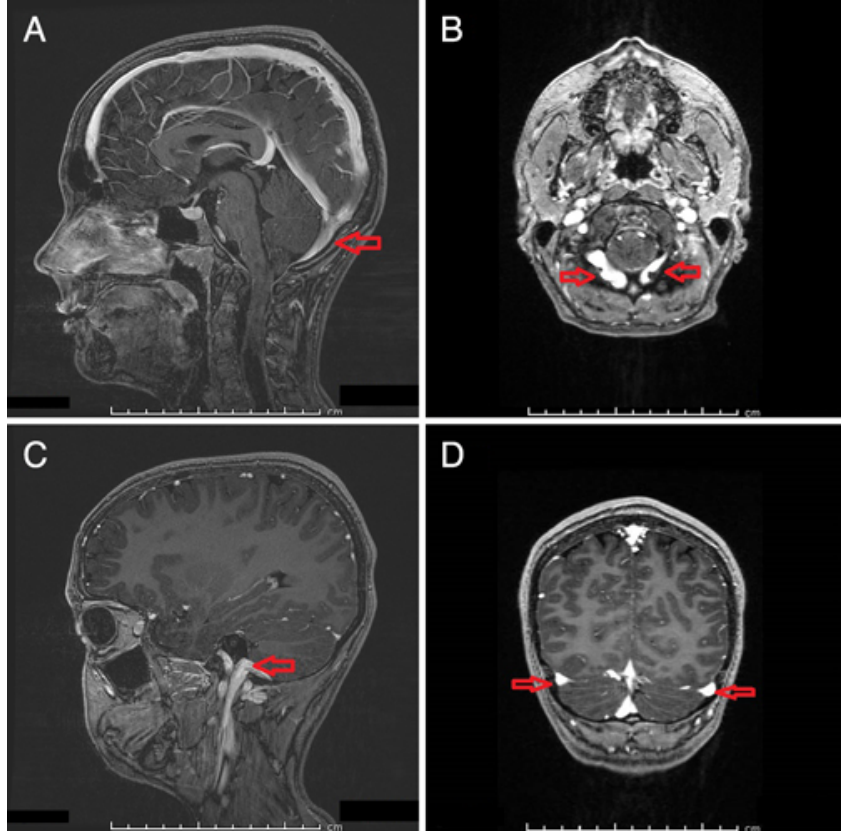

FIG. 4. Preoperative Gd-enhanced T1-weighted sagittal (A and C), axial (B), and coronal (D) MR images showing very prominent occipital sinus and marginal sinuses, which drained directly into the sigmoid sinus and jugular vein bilaterally (arrows). Figure is available in color online only.

now, with only one report of occipital sinus thrombosis leading to a similar picture of intracranial hypertension. ${ }^{1}$ We report on the surgical occlusion of dominant occipital and marginal sinuses leading to intracranial hypertension. This case emphasizes the importance to recognize the importance and drainage pattern of the occipital sinus prior to posterior fossa dura opening. Certain techniques of dura opening with preservation of the occipital sinus have been described ${ }^{6}$ and could be used to avoid such a complication. Such a technique could have been used our case. In patients with CM-I and syrinx, most experts recommend performing durotomy and duraplasty, as this allows microscopic inspection and lysis of arachnoid bands that may be responsible for the syrinx..$^{12}$ However, there is controversy as to whether duraplasty is necessary, particularly in the absence of a syrinx, and many authors perform bone-only decompressions to reduce the risks associated with duraplasty. A multicenter randomized trial is underway to address this. In cases of dominant occipital sinus variant (with a caliber at least equal to or bigger than the transverse sinus and draining into jugular veins), bone-only decompression should be considered in order to avoid this complication, particularly in the absence of a syrinx. In the presence of a patent transverse sinus, our case suggests that CSF drainage and acetazolamide could be effective as the firstline treatment of this condition. We believe this treatment acted as a buffer, alleviating symptoms while the venous outflow could be redirected via the patent transverse sinuses.

\section{Conclusions}

We report a rare case of intracranial hypertension fol- 
lowing surgical occipital and marginal sinus occlusion that was successfully managed with a therapeutic lumbar puncture and acetazolamide. Although occlusion of these sinuses usually bears no clinical consequences, our case illustrates the importance of preoperatively assessing the dural sinus drainage of the posterior fossa and allowing for the possibility of an occipital sinus draining into the jugular bulbs even in the presence of a normal-sized transverse sinus. Preoperative brain imaging including venous drainage visualization and occipital sinus sparing techniques should be considered in the evaluation and avoidance of this condition.

\section{References}

1. Beyrouti R, Mansour M, Kacem A, Zaouali J, Mrissa R: Occipital sinus thrombosis: an exceptional case report. J Stroke Cerebrovasc Dis 25:e71-e73, 2016

2. Dora F, Zileli T: Common variations of the lateral and occipital sinuses at the confluens sinuum. Neuroradiology 20:23-27, 1980

3. Kopuz C, Aydin ME, Kale A, Demir MT, Çorumlu U, Kaya $\mathrm{AH}$ : The termination of superior sagittal sinus and drainage patterns of the lateral, occipital at confluens sinuum in newborns: clinical and embryological implications. Surg Radiol Anat 32:827-833, 2010

4. Lee HC, Lee JY, Ryu SK, Lim JM, Chong S, Phi JH, et al: A method of posterior fossa dural incision to minimize hemorrhage from the occipital sinus: the "mosquito" method. Childs Nerv Syst 32:2429-2431, 2016

5. Okudera T, Huang YP, Ohta T, Yokota A, Nakamura Y, Maehara F, et al: Development of posterior fossa dural sinuses, emissary veins, and jugular bulb: morphological and radiologic study. AJNR Am J Neuroradiol 15:1871-1883, 1994

6. Panigrahi M, Krishnan SS, Varma DR: Crescent posterior fossa durotomy for occipito-marginal venous sinus preservation: a pilot study. Acta Neurochir (Wien) 154:2115-2121, 2012
7. Rhoton AL Jr: The posterior fossa veins. Neurosurgery 47 (3 Suppl):S69-S92, 2000

8. Singh M, Nagashima M, Inoue Y: Anatomical variations of occipital bone impressions for dural venous sinuses around the torcular Herophili, with special reference to the consideration of clinical significance. Surg Radiol Anat 26:480487, 2004

9. Tanoue S, Kiyosue H, Sagara Y, Hori Y, Okahara M, Kashiwagi J, et al: Venous structures at the craniocervical junction: anatomical variations evaluated by multidetector row CT. Br J Radiol 83:831-840, 2010

10. Tubbs RS, Ammar K, Liechty P, Wellons JC III, Blount JP, Salter EG, et al: The marginal sinus. J Neurosurg 104:429431, 2006

11. Widjaja E, Griffiths PD: Intracranial MR venography in children: normal anatomy and variations. AJNR Am J Neuroradiol 25:1557-1562, 2004

12. Zhao JL, Li MH, Wang CL, Meng W: A systematic review of Chiari I malformation: techniques and outcomes. World Neurosurg 88:7-14, 2016

\section{Disclosures}

The authors report no conflict of interest concerning the materials or methods used in this study or the findings specified in this paper.

\section{Author Contributions}

Conception and design: Weil. Acquisition of data: Champagne. Analysis and interpretation of data: Champagne. Drafting the article: Champagne. Critically revising the article: Weil, Cayouette, Lortie, Decarie. Reviewed submitted version of manuscript: all authors. Approved the final version of the manuscript on behalf of all authors: Weil. Study supervision: Weil.

\section{Correspondence}

Alexander G. Weil: Centre Hospitalier Universitaire Sainte-Justine, Montréal, QC, Canada. alexandergweil@gmail.com. 